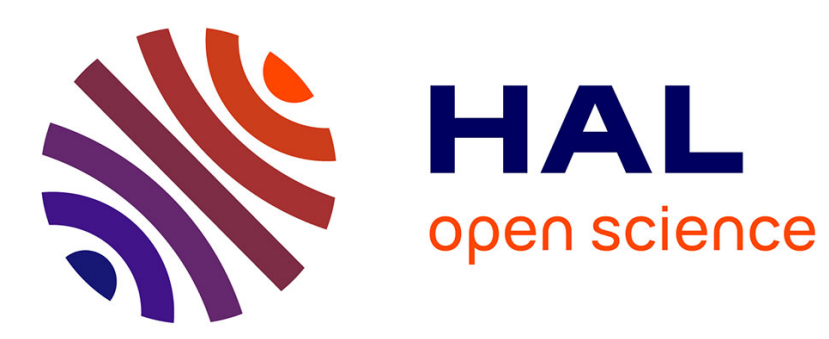

\title{
Shear strength, force distributions and friction mobilization in sheared packings composed of angular particles
}

Emilien Azéma, Nicolas Estrada, Farhang Radjai

\section{To cite this version:}

Emilien Azéma, Nicolas Estrada, Farhang Radjai. Shear strength, force distributions and friction mobilization in sheared packings composed of angular particles. Powder and Grains 2013, 2013, Sydney, Australia. pp.511-514. hal-00842744

HAL Id: hal-00842744

https://hal.science/hal-00842744

Submitted on 9 Jul 2013

HAL is a multi-disciplinary open access archive for the deposit and dissemination of scientific research documents, whether they are published or not. The documents may come from teaching and research institutions in France or abroad, or from public or private research centers.
L'archive ouverte pluridisciplinaire HAL, est destinée au dépôt et à la diffusion de documents scientifiques de niveau recherche, publiés ou non, émanant des établissements d'enseignement et de recherche français ou étrangers, des laboratoires publics ou privés. 


\title{
Shear strength, force distributions and friction mobilization in sheared packings composed of angular particles
}

\author{
Emilien Azéma*, Nicolas Estrada ${ }^{\dagger}$ and Farhang Radjai* \\ ${ }^{*}$ LMGC, Université Montpellier 2-CNRS, Place Eugène Bataillon, 34095 Montpellier cedex 05, France \\ ${ }^{\dagger}$ Departamento de Ingeniería Civil y Ambiental, Universidad de Los Andes, Bogotá, Colombia
}

\begin{abstract}
In this paper, we explore the effect of particle shape angularity on the mechanical behavior of sheared granular packings. A first series of contact dynamics simulations is performed in $2 \mathrm{D}$ with regular polygons with an increasing number of sides ranging from 3 (triangles) to 60. Then, in order to approach "idealized" angular particles, a second series of simulations is performed in 3D with irregular polyhedra with the number of faces ranging from 8 (octahedron-like) to 596. A counterintuitive finding is that the shear strength increases with angularity up to a maximum value and saturates as the particles become more angular (below 6 sides in 2D and 46 faces in 3D). A micromechanical analysis of force and contact orientations, all enhanced by face-face and face-side contacts, reveals that this increase is due to an increase of both contact and force anisotropies, and the saturation for higher angularities is a consequence of a rapid fall-off of the contact and normal force anisotropies compensated by an increase of the tangential force anisotropy.
\end{abstract}

Keywords: Polyhedral particles ; texture ; shear behavior ; contact dynamic method PACS: 45.70.-n,83.80.Fg,61.43.-j

\section{INTRODUCTION}

The role of particle shape for the complex rheology of granular materials is a vast topic, which has recently been addressed both experimentally and numerically $[1,2,3,4,5]$. The existing research results suggest that the effect of shape parameters is often nonlinear and counterintuitive as in the case, for example, of the unmonotonic relation between the elongation of the particles and the packing fraction [6,7]. Nevertheless, a systematic and quantitative investigation of shapedependence is still largely elusive since particle shape characteristics such as elongation, angularity, slenderness or nonconvexity are described by distinct groups of parameters, and the effect of each parameter is not easy to isolate experimentally.

Among various shape characteristics mentioned above, angular shape represents basically a property of polygonal particles in $2 \mathrm{D}$ and polyhedral particles in $3 \mathrm{D}$ $[8,9,10]$. By comparing numerically the shear behavior and microstructure of granular materials composed of pentagonal/polyhedral particles with respect to packings composed of circular/spherical particles, it has been shown that angular particles have higher shear stress and dilatancy $[11,10]$. The origin of these enhanced properties of the angular packings was traced back to the fabric properties in terms of branch vectors and contact forces. In particular, the face-face contacts capture strong force chains leading to a higher force anisotropy.

In this paper, we report on a systematic investigation of the effect of shape angularity by simulating different packings of regular polygons in 2D and irregular polyhedral particles in 3D with different numbers of faces but similar values of their other characteristics. Numerically, the passage from 2D to 3D involves numerical handling of particles of polyhedral shape and a higher numerical efficiency for 3D simulations, but it is necessary in order to approach the behavior of "real" granular media.

\section{NUMERICAL PROCEDURES}

In $2 \mathrm{D}$ with regular polygons, the angularity is simply given by $\alpha=2 \pi / n_{s}$ where $n_{s}$ is the number of sides of the polygons. In contrast, in $3 \mathrm{D}$ a strict procedure need to be defined in order to isolate and control precisely the shape. First, a set of $n_{v}$ vertices are randomly generated on a unit sphere. The convex hull of these points is created by associating three vertices for each face. This condition implies that the number $n_{f}$ of faces is simply given by $n_{f}=2 n_{v}-4$. Secondly, to control the eccentricity of the particles, the degree of distortion $\eta$ from a perfectly spherical shape is taken into account. The parameter $\eta$ is defined as the ratio of the difference of the radii of circumscribed and inscribed spheres on the radius of the circumscribed sphere [12]. For nearly spherical particles we have $\eta<0.1$. In the generation of the verices, the first step is iterated until this condition is satisfied. We define thus the mean angularity $\langle\alpha\rangle$ of a particle as the mean angle between all its adjacent faces. In this simple way, for a given value of $\eta$, we can control the angularity of the particles with a single continuously-variable shape parameter depending only on the number of faces $n_{f}$. 

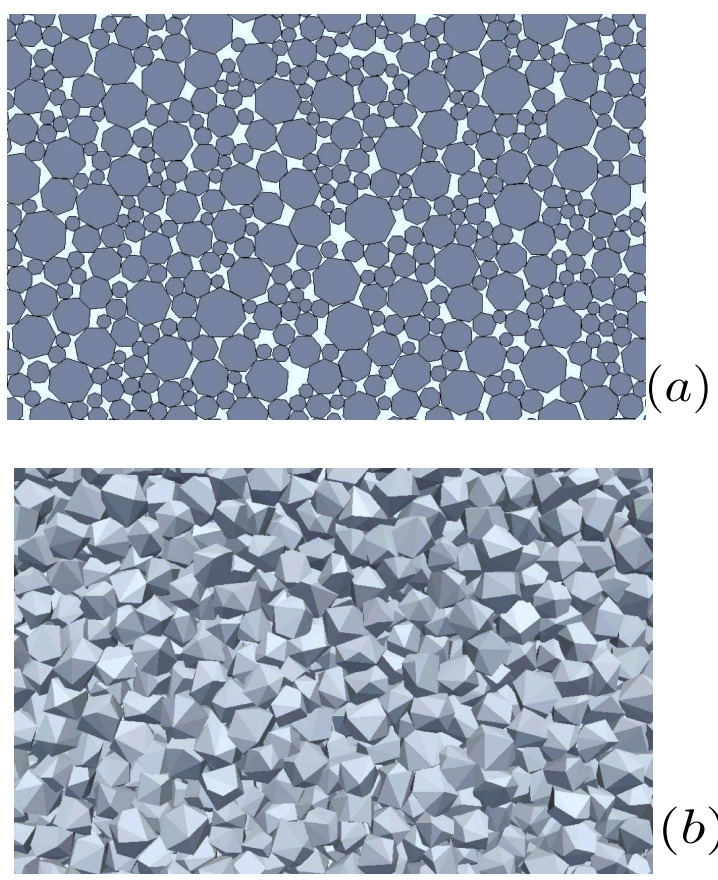

(b)

FIGURE 1. Snapshot of a portion of the 2D and 3D packings at the end of isotropic compression for polygons with $n_{s}=7$ (a) and polyhedra with $n_{f}=20$ (b).

We prepared 13 different packings composed of 10000 regular polygons with the same number of sides $n_{s} \in$ $[3, \ldots, 11,17,30,40,60]$ as well as 7 packings composed of 40000 irregular polyhedra with the same number of faces $n_{f} \in[8,20,30,46,96,176,596]$. Additionally, we build one more packing composed of the same number of disks and spheres respectively. In order to avoid longrange ordering, we introduce a size polydispersity by varying the circumradius of the particles in the range $\left[d_{\min }, 2 d_{\min }\right]$, where $d_{\min }$ is the minimum radius with a uniform distribution by volume fractions.

By means of contact dynamic simulations (CD) [13, $14,15]$ the particles are first compacted by isotropic compression inside a box. The gravity is set to 0 and friction coefficient $\mu$ and $\mu_{w}$ between particles and with the walls, respectively, are set to 0.4 and 0 in order to get homogeneous and isotropic packings. Figure 1 displays snapshots of the packings for $n_{s}=7$ (a) in 2D and $n_{f}=20$ (b) in $3 \mathrm{D}$ at the end of isotropic compaction. These samples are then used as initial configuration for biaxial, respectively triaxial, compression tests. A downward velocity $v_{y}$ is imposed on the upper wall while keeping a constant confining stress on the lateral walls. The strain rate $v_{y} / H$ is low so that the evolution can be considered as quasi-static.

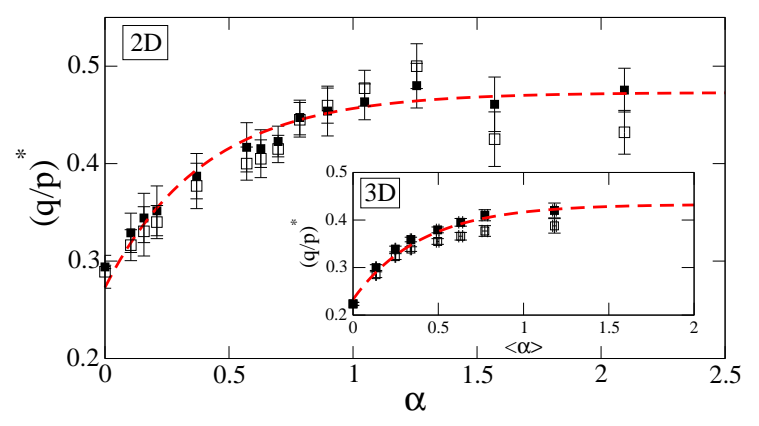

FIGURE 2. Normalized steady state shear stress $(q / p)^{*}$ as a function of particle angularity from raw simulation data (black symbol) and as predicted by Eq.2 (empty symbol) both for 2D and 3D (inset) simulations. The error bars indicate the standard deviation of stress fluctuations in the steady state.

\section{MACROSCOPIC SHEAR STRENGTH}

The stress tensor $\sigma$ can be evaluated from the simulation data as an average over all the contacts of the dyadic product of contact force $\mathbf{f}^{\mathbf{c}}$ and branch vector $\ell^{\mathbf{c}}$ : $\sigma_{\alpha \beta}=n_{c}\left\langle f_{\alpha}^{c} \ell_{\beta}^{c}\right\rangle_{c}$ [16], where $n_{c}$ is the number density of contacts $c$ and the average $\langle\ldots\rangle_{c}$ runs over all contacts in a control volume. In 3D with axial symmetry we define the stress deviator $q=\left(\sigma_{1}-\sigma_{3}\right) / 3$ and the mean stress $p=\left(\sigma_{1}+\sigma_{2}+\sigma_{3}\right) / 3$, where $\sigma_{1,2,3}$ are the principal stress values. In $2 \mathrm{D}$, we set $q=\left(\sigma_{1}-\sigma_{2}\right) / 2$ and $p=\left(\sigma_{1}+\sigma_{2}\right) / 2$. For our system of perfectly rigid particles, the stress state is characterized by the mean stress $p$ and the normalized shear stress $q / p$ [17]. During shear, the shear stress jumps initially to a high value before decreasing to a nearly constant value in the steady state. The steady-state shear stress $(q / p)^{*}$ characterizes the shear strength of the material.

Figure 2 shows the evolution of $(q / p)^{*}$ as a function of angularity $\alpha$ of the particles. The shear strength first increases with $\alpha$ from 0.29 in 2D and 0.21 in 3D and then saturates for particles having a less number of sides (or faces in 3D) for which $(q / p)^{*} \simeq 0.47$ in $2 \mathrm{D}$ and 0.43 in 3D. The data are well fit to an exponential function (red dashed line). The fast increase of $(q / p)^{*}$ with $\alpha$ and its saturation is rather unexpected as it indicates that small deviations of the shape from disk or sphere have stronger effect on the value of $(q / p)^{*}$ than the larger variations of angularity for low numbers of sides (or faces).

\section{TEXTURE AND FORCE ANISOTROPIES}

Figure 3 shows a typical map of normal forces in our 2D and 3D configurations. The force chains are clearly inhomogeneous forming anisotropic structures with a "zigzag" shape. The most basic descriptor of anisotropy is 


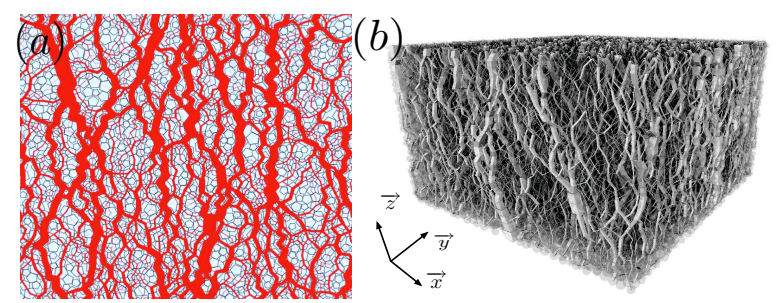

FIGURE 3. Snapshots of normal forces in packings composed of polygons with $n_{s}=5$ (a) and polyhedra with $n_{f}=$ 30(b) in the steady state.

the probability distribution $P(\mathbf{n})$ of the contact normals, which is generically nonuniform. The unit vector $\mathbf{n}$ is described by a single angle $\theta$ in 2D and by azimuthal and longitudinal angles $(\theta, \phi)$ in $3 \mathrm{D}$. Assuming axial symmetry for 3D simulations, which is the case for our triaxial test, the probability density $P(\theta)$ of contact orientations $\theta$ provides the required statistical information about the contact network.

A local frame $(\mathbf{n}, \mathbf{t})$ can be attached to each contact, where $\mathbf{t}$ is an orthonormal unit vector. The local geometry associated with the two contact neighbors is characterized by the branch vector $\ell$ joining the particle centers. It can be projected in the local contact frame $\ell=\ell_{\mathbf{n}} \mathbf{n}+\ell_{\mathbf{t}} \mathbf{t}$. Note that, in contrast to circular particles, for which $\ell_{t}=0$, in a packing of polygonal or polyhedral particles this component is nonzero. In the same way, the contact force $\mathbf{f}$ can be expressed in terms of its normal and tangential components: $\mathbf{f}=\mathbf{f}_{\mathbf{n}} \mathbf{n}+\mathbf{f}_{\mathbf{t}} \mathbf{t}$.

Along with $P(\theta)$, the anisotropy of the packing can be further characterized by the angular averages of the components of the branch vectors and contact forces as a function of the orientation $\theta:\left\langle\ell_{n}\right\rangle(\theta),\left\langle\ell_{t}\right\rangle(\theta),\left\langle f_{n}\right\rangle(\theta)$ and $\left\langle f_{t}\right\rangle(\theta)$. These functions describe the general state of anisotropy, and both experiments and simulations show that, under shearing, the packing self-organizes into a state where a simple approximation based on spherical harmonics at leading terms captures their anisotropies $[18,10]$ :

$$
\left\{\begin{array}{l}
P_{\theta}(\theta)=\frac{1}{4 \pi}\left\{1+a_{c}\left[3 \cos ^{2}\left(\theta-\theta_{c}\right)-1\right]\right\} \\
\left\langle\ell_{n}\right\rangle(\theta)=\frac{1}{4 \pi}\left\{1+a_{\ell n}\left[3 \cos ^{2}\left(\theta-\theta_{\ell n}\right)-1\right]\right\}, \\
\left\langle f_{t}\right\rangle(\theta)=\left\langle\ell_{n}\right\rangle a_{\ell t} \sin 2\left(\theta-\theta_{\ell t}\right) \\
\left\langle f_{n}\right\rangle(\theta)=\left\langle f_{n}\right\rangle\left\{1+a_{f n}\left[3 \cos ^{2}\left(\theta-\theta_{f n}\right)-1\right]\right\} \\
\left\langle f_{t}\right\rangle(\theta)=\left\langle f_{n}\right\rangle a_{f t} \sin 2\left(\theta-\theta_{f t}\right),
\end{array}\right.
$$

where $a_{c}$ is the contact orientation anisotropy, $a_{\ell n}$ and $a_{\ell t}$ are the normal and tangential branch anisotropies, $a_{f n}$ and $a_{f t}$ are the normal and tangential force anisotropies. The angles $\theta_{c}, \theta_{\ell n}, \theta_{\ell t}, \theta_{f n}$, and $\theta_{f t}$ are the corresponding privileged directions. These directions can all be different, but they coincide with the principal stress direction $\theta_{\sigma}$ in a sheared granular material $[18,10,7]$. A simi-
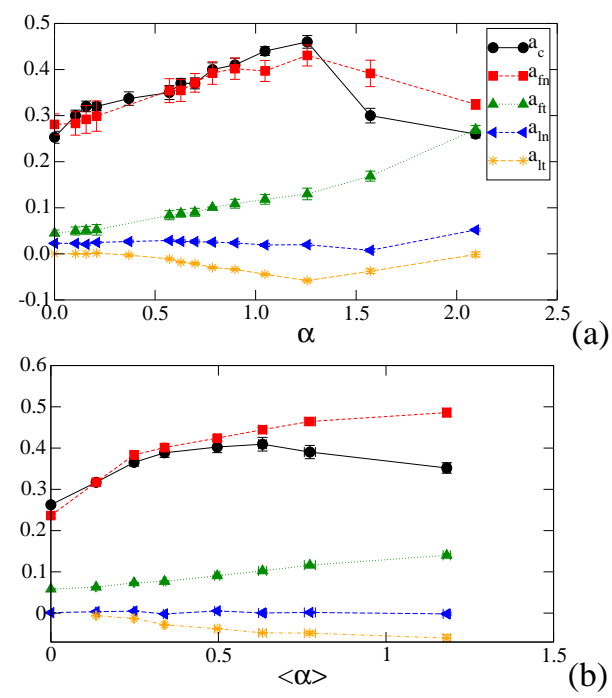

FIGURE 4. Evolution of the anisotropy parameters $a_{c}, a_{\ell n}$, $a_{\ell t}, a_{f n}$ and $a_{f t}$ as a functions of the particle shape angularity for $2 \mathrm{D}(\mathrm{a})$ and $3 \mathrm{D}(\mathrm{b})$ simulations. Error bars represent the standard deviation in the steady state.

lar relation is obtained in 2D by replacing the function $3 \cos ^{2}(\theta)-1$ by $\cos (2 \theta)$ and $1 /(4 \pi)$ by $1 /(2 \pi)$.

The anisotropies $a_{c}, a_{\ell n}, a_{\ell t}, a_{f n}$ and $a_{f t}$ are interesting descriptors of granular microstructure and force transmission properties because they underlie the different microscopic origins of shear strength. Indeed, it can be shown that the general expression of the stress tensor leads to the following simple relation $[18,10]$ :

$$
\frac{q}{p} \simeq \begin{cases}\frac{1}{2}\left(a_{c}+a_{\ell n}+a_{\ell t}+a_{f n}+a_{f t}\right) & 2 \mathrm{D} \\ \frac{5}{2}\left(a_{c}+a_{\ell n}+a_{\ell t}+a_{f n}+a_{f t}\right) & 3 \mathrm{D} .\end{cases}
$$

where the cross products between the anisotropy parameters have been neglected. The predicted values of $(q / p)^{*}$ by this equation are shown in Fig. 2 (empty symbol) together with the measured values as a function of $\alpha$. We see that Eq. 2 approximates well the shear strength for all values of $\alpha$ both in 2D and 3D.

The evolution of the five anisotropies with $\alpha$ is shown in Fig. 4. The normal and tangential branch anisotropies, $a_{\ell n}$ and $a_{\ell t}$, are negligible in comparison to the other anisotropy parameters. This is due to the absence of shape eccentricity of the particles [7] and to the low span in the particle size distribution [19].

The other anisotropies, $a_{c}, a_{f n}$, and $a_{f t}$, grow as $\alpha$ increases from zero (for the disk/sphere packing) up to $\alpha \simeq 1.25$ in $2 \mathrm{D}$ and 0.8 in $3 \mathrm{D}$. This increase of all anisotropies underlies the observed increase of the shear strength in this range. The increase of the anisotropies reflects the increasing number of side-side contacts (faceface and face-side in 3D), which capture the strong force 

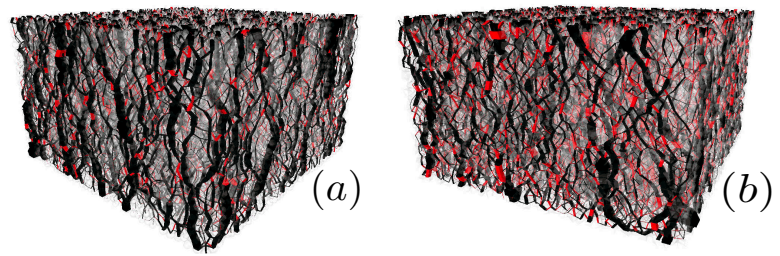

FIGURE 5. Snapshot of radial forces in packing for $n_{f}=$ 176(a) and $n_{f}=20$ (b) in the steady state. Line thickness is proportional to the radial force. In red, the force where $f_{t}=\mu f_{n}$

chains and form column-like structures, which can be stable without sidewise support $[11,10,20]$.

Then, at higher level of angularity a rapid decrease of $a_{c}$ (and $a_{n}$ for 2D) occurs whereas $a_{f t}$ grows at the same time. As it is observed in Fig. 4, this increase of $a_{f t}$ is large enough to compensate additively (See Eq. (2)) the decrease of $a_{c}$ (and $a_{f n}$ in 2D), so that the shear strength remains nearly constant, as observed in Fig. 2. This happens because very angular particles also have very few faces, so that it becomes difficult to orient these faces perpendicularly to the direction in which the forces are being transmitted. This causes the anisotropies $a_{c}$ to decrease. In addition, $a_{f t}$ increases, since the stability of such contacts relies on a strong activation of friction forces $[11,10,20]$. This is well illustrated in Fig. 5 where a map of mobilized forces (ie $f_{t}=\mu f_{n}$ ) is shown in red for $n_{f}=176(\mathrm{a})$ and $n_{f}=20(\mathrm{~b})$.

\section{CONCLUSIONS}

In this paper, we investigated the effect of particle shape angularity in 2D and 3D for the quasistatic behavior of sheared granular materials by means of contact dynamics simulations. We considered a first series of packings composed of regular polygons as an "idealized" material. In order to approach the behavior of "real" angular materials a second series of simulations were performed in $3 \mathrm{D}$ with irregular polyhedral particles. The macroscopic and microstructural properties of several packings composed of a large number of particles were analyzed as a function of the angularity of the particles in the steady state.

It was shown that the shear strength first increases with the angularity and then remains nearly constant at larger angularity. Interestingly, we evidenced that the increase of all anisotropies underlies the observed increase of the shear strength whereas the plateau of shear strength results from a decrease of the contact anisotropies compensated by an increase of the tangential force anisotropy. This transition results from a geometric effect that becomes dominant for very angular particles (a few number of faces along the stress direction) and that implies that the stability of the packing relies strongly on friction forces.

Nevertheless, much more work is needed in order to understand the mechanical role of each contact type on the stress transmission. An idea is to isolate the contribution of each contact on the texture and forces anisotropies. Another well known feature of idealized granular media is that the contacts can be classified into strong and weak networks with distinct mechanical roles. It would be interesting to revisit this concept for packings composed of angular particles.

We acknowledge financial support from the EcosNord program (Grant No. C12PU01).

\section{REFERENCES}

1. K. Mair, K. Frye, and C. Marone, Journal Of Geophysical Research 107 (2002).

2. F. Alonso-Marroquin, and H. J. Herrmann, Phys. Rev. E 66, 021301 (2002).

3. Y. Guo, and J. Morgan, Journal Of Geophysical Research 109 (2004).

4. J. L. Anthony, and C. Marone, Journal Of Geophysical Research 110 (2005).

5. S. A. Galindo-Torres, J. D. Munoz, and F. AlonsoMarroquin, physical review E 82, 056713 (2010).

6. A. Donev, F. Stillinger, P. Chaikin, and S. Torquato, Phys Rev Lett. 92, 255506-1, 255506-4 (2004).

7. E. Azéma, and F. Radjaï, Phys Rev E 81, 051304 (2010).

8. E.-G. Nezami, Y. Hashash, D. Zaho, and J. Ghaboussi, Computers and Geotechnics 31, 575-587 (2004).

9. G. Saussine, C. Cholet, P. Gautier, F. Dubois, C. Bohatier, and J. Moreau, Comput. Methods Appl. Mech. Eng. 195, 2841 - 2859 (2006).

10. E. Azéma, F. Radjai, and G. Saussine, Mechanics of Materials 41, 721-741 (2009).

11. E. Azéma, F. Radjai, R. Peyroux, and G. Saussine, Phys. Rev. E 76, 011301 (2007).

12. Cegeo, B. Saint-Cyr, K. Szarf, C. Voivret, E. Azéma, V. Richefeu, J.-Y. Delenne, G.Combe, C. NouguierLehon, P. Villard, P. Sornay, M. Chaze, and F. Radjai, Eur. Phys. Letter p. 5 (2012).

13. J. Moreau, "An introduction to unilateral dynamics," in Novel approaches in civil engineering, edited by M. Frémond, and F. Maceri, Springer-Verlag, Berlin, 2004, vol. 14 of Lecture Notes in Applied and Computational Mechanics, pp. 1-46.

14. F. Radjai, and F. Dubois, editors, Discrete Numerical Modeling of Granular Materials, Wiley-ISTE, New-York, March 2011, iSBN: 978-1-84821-260-2.

15. www.lmgc.univ-montp2.fr/LMGC90.

16. J. Moreau, European J. Mech. A Solids 13, 93-114 (1994).

17. J. Mitchell, and K. Soga, Fundamentals of Soil Behavior, Wiley, New-York, NY, 2005.

18. L. Rothenburg, and R. J. Bathurst, Geotechnique 39, 601-614 (1989).

19. C. Voivret, F. Radjai, J.-Y. Delenne, and M. S. E. Youssoufi, Phys. Rev. Lett. 102, 178001 (2009).

20. E. Azéma, N. Estrada, and F. Radjai, Physica Reviews E 86, 041301 (2012). 